Association for Information Systems AIS Electronic Library (AISeL)

Wirtschaftsinformatik Proceedings 1999

Wirtschaftsinformatik

February 1999

\title{
Internet Bill Presentment and Payment als neue Form des Electronic Billing
}

Stefan Eicker

Universität GH Essen, eicker@wi-inf.uni-essen.de

Holger Schwichtenberg

Universität GH Essen, holger.schwichtenberg@uni-essen.de

Follow this and additional works at: http://aisel.aisnet.org/wi1999

\section{Recommended Citation}

Eicker, Stefan and Schwichtenberg, Holger, "Internet Bill Presentment and Payment als neue Form des Electronic Billing" (1999). Wirtschaftsinformatik Proceedings 1999. 10.

http://aisel.aisnet.org/wi1999/10

This material is brought to you by the Wirtschaftsinformatik at AIS Electronic Library (AISeL). It has been accepted for inclusion in Wirtschaftsinformatik Proceedings 1999 by an authorized administrator of AIS Electronic Library (AISeL). For more information, please contact elibrary@aisnet.org. 


\section{Internet Bill Presentment and Payment als neue Form des Electronic Billing}

\section{Stefan Eicker}

Universität GH Essen (Eicker@wi-inf.uni-essen.de)

Holger Schwichtenberg

Universität GH Essen (ㅂolger.Schwichtenberg@uni-essen.de)

\section{Inhalt}

1 Einleitung

2 Grundlagen der Rechnungsstellung

3 Bestehende Billing-Verfahren

4 Internet Bill Presentment and Payment (IBPP)

4.1 Web-Billing

4.2 E-Mail-Billing

4.3 Integration von Web- und E-Mail-Billing

4.4 Indirektes Internet Bill Presentment and Payment

4.5 Rechtsfragen des Electronic Billing

5 Konzeption eines IBPP-Systems für ein Telekommunikationsunternehmen

5.1 Architektur

5.2 Bill Presentment der Telekommunikationsrechnung 


\begin{abstract}
In einem Projekt wurde für ein großes Telekommunikationsunternehmen die Möglichkeit untersucht, das Internet für den Versand und die Bezahlung von Rechnungen zu nutzen. Der vorliegende Beitrag faßt die Ergebnisse der Untersuchung zusammen: Aufbauend auf einer Betrachtung der Grundlagen der Rechnungsstellung und bestehender Billing-Verfahren präsentiert er das Web-Billing, das E-Mail-Billing und die Bill Consolidation als Lösungsansätze für das Internet Bill Presentment and Payment (IBPP). Die Ansätze werden insbesondere im Hinblick auf die Erfüllung der Anforderungen von Rechnungssteller und Rechnungsempfänger sowie auch aus juristischer Sicht diskutiert. Aus der für das Telekommunikationsunternehmen entwickelten Konzeption für ein IBPP-System stellt der Beitrag die Systemarchitektur sowie das Bill Presentment der Telekommunikationsrechnung vor.
\end{abstract}

\title{
1 Einleitung
}

Allein in den USA werden von Unternehmen im Jahr 27 Milliarden Rechnungen (21 Milliarden im Business-To-Consumer- und 6 Milliarden im Business-ToBusiness-Bereich) versandt, um das Geld für erbrachte Leistungen einzufordern (Craft/Johnson 1998, S. 5-6). Traditionell werden Rechnungen auf Papier erstellt und auf dem Postweg befördert; auch die Zahlung der Rechnungen ist heute noch teilweise an Papierbelege gebunden. Zwar existiert im Business-To-Business-Bereich in Gestalt von Verfahren des Electronic Data Interchange (EDI) bereits seit einigen Jahren eine technische Lösung zum elektronischen Rechnungsversand. Genutzt wird sie jedoch vergleichsweise selten, zumeist in branchenspezifischen Anwendungen.

Im folgenden wird der Ansatz vorgestellt, das Internet für die Präsentation und den Versand von Rechnungen zu nutzen. Dazu werden zunächst kurz die Grundlagen der Rechnungsstellung, d.h. die Aufgabe der Rechnung, der Rechnungsproze $\beta$ und die Anforderungen an den Prozeß, diskutiert. Darauf aufbauend zeigt der Beitrag die Nachteile der bestehenden papierbasierten sowie der elektronischen Billingverfahren auf. Mit dem Web-Billing, dem E-Mail-Billing und der Bill Consolidation werden dann Ansätze für das Internet Bill Presentment and Payment (IBPP) präsentiert; angesprochen werden insbesondere auch bestehende rechtliche Problemfelder. Schließlich stellt der Beitrag aus der Konzeption eines IBPP-Systems, die für ein großes Telekommunikationsunternehmen entwickelt wurde, die Systemarchitektur sowie das Bill Presentment der Telekommunikationsrechnung vor. 


\section{Grundlagen der Rechnungsstellung}

Eine Rechnung erfüllt drei Funktionen: die Zahlungsaufforderungsfunktion, die Leistungsnachweisfunktion und die Marketingfunktion. Die Zahlungsaufforderungsfunktion besteht darin, daß der Rechnungssteller durch eine Rechnung erklärt, daß er seinen Teil des Vertrages (Lieferung eines Produktes oder einer Dienstleistung) erfüllt hat. Er fordert durch die Rechnung seinen Vertragspartner (den Kunden) auf, die seinerseits im Vertrag zugesagte Leistung, die Bezahlung, zu erbringen. Der Kunde kann mit der Rechnung später gegenüber Dritten (insbesondere den Finanzbehörden) nachweisen, daß von ihm eine Zahlung verlangt wurde.

Durch die Rechnung dokumentiert der Rechnungssteller außerdem die von ihm erbrachte Leistung, insbesondere dann, wenn es keinen separaten Lieferschein gibt. Diese Leistungsnachweisfunktion erfüllt die Rechnung durch die Auflistung beispielsweise von Einzelpositionen bei einer Produktbestellung, von geleisteten Arbeitsstunden bei einer Dienstleistung oder der Einzelverbindungen bei einer Telekommunikationsrechnung. Der Rechnungsempfänger benötigt die Informationen zur Rechnungsprüfung sowie eventuell für seine interne Kostenrechnung, insbesondere zur Verteilung auf Kostenstellen.

Schließlich ist die Rechnung nicht zuletzt für den Rechnungssteller auch ein Kommunikations- und Informationsinstrument. Der Rechnungssteller kann das Instrument nutzen, um durch After-Sales-Promotion kognitive Dissonanzen beim Kunden zu reduzieren (Nieschlag/Dichtl/Hörschgen 1988， S. 460 ff.; Geml/Geisbüsch/Lauer, S. 144) oder um auf dem Wege des Cross-Selling dem Kunden weitere Produkte und Dienstleistungen anzubieten (Geml/Geisbüsch/Lauer, S. 63 f.). Diese Marketingfunktion der Rechnung ist gerade in den Branchen (z.B. der Telekommunikation) von besonderer Bedeutung, in denen Rechnungen zwar relativ häufig verschickt werden, der direkte Kontakt zum Kunden aber nur selten besteht.

Abbildung 1 zeigt ein vereinfachtes Prozeßdiagramm der Rechnungsstellung. Der Prozeß kann in drei Teilprozesse unterteilt werden (Open Financial Exchange 1998a; CyberCash 1998, COBWEB 1998), das Bill Presentment, das Bill Payment und das Bill Posting. Bill Presentment umfaßt die Rechnungsübermittlung vom Rechnungsaussteller an den Kunden. Bill Payment bezieht sich auf die Bezahlung der Rechnung durch den Kunden. Bill Posting beinhaltet die Übermittlung der Zahlungsdaten an den Rechnungssteller und den Import der Daten in seine internen Billing-Systeme (z.B. zum Abgleich des Kundenkontos in der Debitorenbuchhaltung).

Tabelle 1 schließt die einführende Betrachtung der Rechnungsstellung ab, indem sie die wesentlichen Anforderungen zusammenfaßt, die aus der Sicht des Rechnungsstellers einerseits und des Rechnungsempfängers andererseits an den Rechnungsprozeß zu stellen sind. 


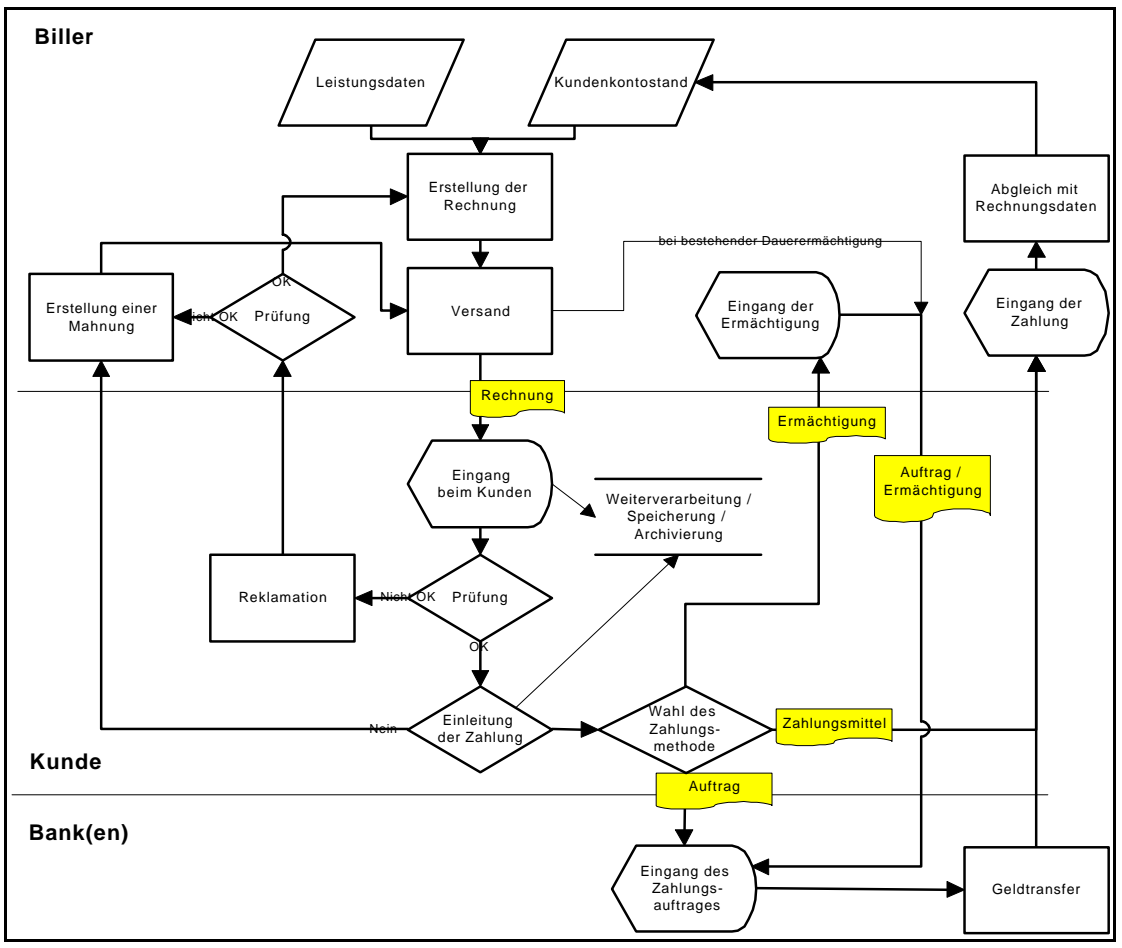

Abbildung 1: Der Rechnungsprozeß

\begin{tabular}{|c|c|c|}
\hline & $\begin{array}{l}\text { Anforderungen aus der Sicht } \\
\text { des Rechnungsstellers }\end{array}$ & $\begin{array}{l}\text { Anforderungen aus der Sicht } \\
\text { des Rechnungsempfänger }\end{array}$ \\
\hline $\begin{array}{l}\text { Bereich } \\
\text { Bill Pre- } \\
\text { sentment }\end{array}$ & $\begin{array}{l}\text { 1) Niedrige Kosten } \\
\text { 2) Hohe Geschwindigkeit } \\
\text { 3) Zuverlässigkeit } \\
\text { 4) Nachweisbarkeit des Rech- } \\
\text { nungszugangs } \\
\text { 5) Datenschutz } \\
\text { 6) Einsatz der Rechnung als Mar- } \\
\text { ketinginstrument } \\
\text { 7) Minimierung des Aufwandes } \\
\text { für das Customer Care }\end{array}$ & $\begin{array}{l}\text { 1) Einfache Zugänglichkeit } \\
\text { 2) Verständlichkeit } \\
\text { 3) Genauigkeit } \\
\text { 4) Auswertbarkeit } \\
\text { 5) Weiterverarbeitbarkeit } \\
\text { 6) Archivierbarkeit } \\
\text { 7) Guter Support } \\
\text { 8) Datenschutz } \\
\text { 9) Steuerrechtliche Anerkennung } \\
\text { 10) Niedrige Kosten }\end{array}$ \\
\hline $\begin{array}{l}\text { Bereiche } \\
\text { Bill Pay- } \\
\text { ment und } \\
\text { Bill Po- } \\
\text { sting }\end{array}$ & $\begin{array}{l}\text { 8) Einfache Zahlungssysteme } \\
\text { 9) Konsistenz zwischen Rech- } \\
\text { nungsdaten und Zahlungsdaten } \\
\text { 10) Effizientes Bill Posting }\end{array}$ & $\begin{array}{l}\text { 11) Komfortable Zahlungsschnitt- } \\
\text { stelle } \\
\text { 12) Übernahme der Rechnungs- } \\
\text { daten } \\
\text { 13) Einfluß auf den Zeitpunkt der } \\
\text { Zahlung }\end{array}$ \\
\hline
\end{tabular}

Tabelle 1: Übersicht über die Anforderungen an den Rechnungsprozeß 


\section{Bestehende Billing-Verfahren}

Ein Rechnungssteller verwaltet die Abrechnungsdaten üblicherweise in einem EDV-System (Billing-System). Er druckt die Daten als Rechnung auf Papier aus und schickt sie dem Kunden zu. Für die Zustellung der Rechnung wird traditionell die konventionelle Briefpost verwendet. Beim Kunden müssen die Daten der Rechnung zur Weiterverarbeitung in EDV-Systemen erneut erfaßt werden. Deshalb verwundert es nicht, daß schätzungsweise $70 \%$ aller geschäftlichen Daten, die in Computer eingegeben werden, von einem anderen Computer ausgegeben wurden (Soetzer 1994, S. 16).

Beim Bill Payment und Bill Posting werden ebenfalls häufig Papierbelege ausgetauscht; Beispiele sind Überweisungsträger, Verrechnungsschecks und Kontoauszüge. Eine Vorstellung von der Anzahl dieser versendeten Papierbelege geben entsprechende Untersuchungen in den USA. Dort werden 90\% aller Rechnungen - also etwa 24 Milliarden Rechnungen - mit einem per Post verschickten Verrechnungsscheck bezahlt (Orr 1998; Dittrich 1998).

Das papierbasierte Billing kann offensichtlich einige Anforderungen an den Rechnungsprozeß nicht erfüllen. Aus der Sicht des Rechnungsstellers ist:

- der Druck, die Kuvertierung und der Versand der Rechnungen teuer und langsam;

- die Nachweisbarkeit des Zugangs nicht gegeben (außer bei der Verwendung sehr teurer Briefzusatzleistungen wie Einschreiben und Rückschein);

- der Einsatz der Rechnung als Marketinginstrument wegen der hohen Zusatzkosten für Beilagen eingeschränkt;

- die Konsistenz zwischen Rechnung und Zahlungsdaten nur sichergestellt, wenn der Kunde vom Rechnungssteller mitgelieferte Zahlungsvordrucke verwendet;

- ein maschinelles Bill Posting erschwert, wenn die Zahlungsdaten auf Papierbelegen eingehen.

Aus der Sicht des Kunden ist:

- die Zugänglichkeit der Rechnung bei Abwesenheit vom Wohnsitz erschwert;

- die Darstellung der Rechnung nicht an individuelle Bedürfnisse anpaßbar;

- die Auswertung, die die Rechnung liefert, häufig unzureichend;

- die Weiterverarbeitbarkeit durch den Medienbruch aufwendig und fehleranfällig;

- die Archivierung der Papierbelege aufwendig.

Zur Vermeidung der Nachteile der papierbasierten Rechnungszustellung und -bezahlung wurden Electronic Billing-Verfahren entwickelt. Sie sind allerdings alle mit dem Nachteil behaftet, daß sie das Bill Presentment und das Bill Payment nur isoliert voneinander abdecken.

Verfahren des Electronic Data Interchange (EDI) sehen Datenformate zum Electronic Bill Presentment vor (Stahlknecht 1993, S. 396). Allerdings hat sich EDI aufgrund der hohen Kosten für die Einführung und aufgrund der zögerlichen 
Durchsetzung von Standards wie EDIFACT nur in wenigen Branchen etabliert. Für den Rechnungsversand im Business-To-Consumer-Bereich sind die bisherigen EDI-Ansätze aufgrund ihrer Komplexität grundsätzlich ungeeignet.

Für das Electronic Bill Payment und Bill Posting sind sog. Electronic Funds Transfer Systems (EFT-Systeme) entwickelt worden (Illingworth 1997, S. 162). Beispiele für EFT-Systeme im Privatkundenbereich sind das Homebanking (über T-Online, das Internet oder Direkteinwahl) sowie Geldautomaten mit Zahlungsfunktion. Die Systeme sehen allerdings keine Möglichkeit zum elektronischen Rechnungsempfang vor. Eine Ausnahme bildet nur das Geldautomatennetz der Bank of Ireland, das das Betrachten und Bezahlen von Rechnungen am Automat für die Kreditkartenrechnungen der Bank sowie für sechs weitere Rechnungssteller anbietet (O'Sullivan 1998, S. 52).

Im Geschäftskundenbereich ermöglicht das in den 70er Jahren von den deutschen Banken konzipierte Datenträger-Austauschformat (DTA), elektronische Zahlungsdaten zwischen Bank und Kunde auszutauschen (Zwischenberger 1998). Das DTA-Format weist allerdings einige Defizite auf; insbesondere sieht das Format keinen strukturierten Nachrichtenteil für den Verwendungszweck vor; außerdem ist es mit einer Länge von 13x27 Stellen den heutigen Anforderungen im kommerziellen Zahlungsverkehr nicht mehr gewachsen (Zwischenberger 1998).

Zusammenfassend ist somit festzustellen, daß die bestehenden Electronic BillingVerfahren die Anforderungen, die an den Rechnungsprozeß zu stellen sind, nur zu einem Teil erfüllen.

\section{Internet Bill Presentment and Payment (IBPP)}

Der Versand und die Bezahlung einer Rechnung über das Internet bietet gegenüber den bestehenden Electronic Billing-Verfahren den wesentlichen Vorteil, daß mit dem Internet ein kostengünstiges Transportmedium benutzt wird, das auch Privathaushalten zur Verfügung steht. Die Bedienung des Internets ist vergleichsweise einfach und einem großen, rasch weiter wachsenden Personenkreis bekannt. Durch die weltweite Verfügbarkeit besteht zudem für einen Rechnungsempfänger ein Zugang zu seinen Rechnungen unabhängig von seinem Aufenthaltsort.

Im folgenden werden zunächst das Web-Billing und das E-Mail-Billing sowie die Möglichkeit der Integration der beiden Ansätze betrachtet. Anschließend wird mit der Bill Consolidation die Alternative des indirekten Internet Bill Presentment und Payment vorgestellt, bei der zwischen Rechnungssteller und Rechnungsempfänger eine dritte, vermittelnde Instanz gesetzt wird. Schließlich werden Rechtsfragen des Electronic Billing diskutiert. 


\subsection{Web-Billing}

Als "Web-Billing" wird hier die Übertragung der Rechnung über das World Wide Web (WWW) bezeichnet. Die Übertragung der Rechnung ("Web-Bill") erfolgt in einem vom Browser darstellbaren Format (z.B. HTML oder XML) oder auch in einem Format, das von einer Helper-Application verarbeitet werden kann. Beispiele für Helper-Applikationen sind z.B. DBase, Microsoft Excel und Personal Finance Management-Software wie Microsoft Money oder Intuit Quicken. Der Rechnungsempfänger löst nach der Prüfung der Rechnung die Zahlung aus; die zugehörigen Daten werden ebenfalls über das WWW übertragen.

Das WWW bietet durch seine Interaktivität dem Rechnungsempfänger die Möglichkeit, die Art der Rechnungsdarstellung selbst zu gestalten: Beispiele sind die Auswahl der Sprache, der Präsentationsform (Text oder Graphik), der Schriftarten, der Farben etc. Darüber hinaus kann die Rechnung in verschiedene, durch Hyperlinks verknüpfte Ebenen unterteilt sein, so daß der Rechnungsempfänger den Detaillierungsgrad seiner Sicht auf die Rechnung selbst bestimmen kann. Außerdem können umfangreiche konfigurierbare Auswertungsmöglichkeiten der Rechnung (z.B. Auswertung der Leistungsbeanspruchung nach Tagen) bereitgestellt werden.

Für die maschinelle Weiterverarbeitbarkeit der Rechnung ist HTML aufgrund mangelnder Strukturierung und fehlender Semantik nicht geeignet. Mit Open Financial Exchange (OFX), einem XML-ähnlichen Konzept (Open Financial Exchange 1998b), und dem GOLD Message Standard (Integrion Financial Network 1997) existieren bereits zwei Vorschläge zur Strukturierung von Rechnungsdaten. Außerdem können Rohdaten über die erbrachten Leistungen per HTTP oder FTP zum Kunden übertragen und von ihm mit Hilfe eigener oder durch den Rechnungssteller bereitgestellter Software ausgewertet werden.

Als Endgeräte kommen beim Web-Billing nicht nur PCs, sondern auch andere internetfähige Geräte wie Web-Telefone und Web-TV-Geräte in Frage. Grundsätzlich könnte das Web-Billing auch in die Funktionalität von Geldautomaten integriert werden (O’Sullivan 1998, S. 55). Damit wären elektronische Rechnungen auch für Kunden zugänglich, die noch über keinen Internetzugang verfügen.

Im Rahmen des Web-Billing sind das Bill Presentment und das Bill Payment vollständig miteinander verknüpfbar, wobei insbesondere die Konsistenz zwischen den Rechnungsdaten und den Zahlungsdaten automatisch sichergestellt werden kann. Außerdem kann das Web-Billing Rechnungsempfängern Funktionen zum Scheduling von Zahlungen anbieten. Der Rechnungsempfänger ist hierbei nicht auf ein Zahlungssystem festgelegt, sondern kann aus den im Internet verfügbaren EFT-Systemen - z.B. in Abhängigkeit von Rechnungshöhe und Transaktionskosten - das geeignete System auswählen.

Dem Rechnungssteller bietet das Web-Billing die Gelegenheit, Marketinginstrumente einzusetzen, wenn die Kunden auf seine WWW-Site zugreifen. Dadurch, 
daß der Kunde sich auf der Site authentifizieren muß, kann der Rechnungssteller insbesondere den Kunden eindeutig identifizieren und ein gezieltes und erfolgversprechendes One-To-One-Marketing betreiben. Dabei stehen ihm die vielfältigen multimedialen Möglichkeiten des WWW zur Verfügung. Außerdem können durch das Web-Billing auch viele Aufgaben des Customer Care effizienter gestaltet werden, indem internettypische Hilfesysteme wie Frequently Asked Questions (FAQ), Foren oder Wissensdatenbanken eingesetzt werden.

Den Vorteilen des Web-Billing steht aus der Sicht des Rechnungsempfängers allerdings ein gravierender Nachteil gegenüber: Da das WWW ein Pull-Dienst ist, wird der Rechnungsempfänger nicht über den Eingang einer Rechnung informiert. Er muß somit selbst aktiv werden und - da er die Rechnungen nicht wie beim papierbasierten Billing an einem zentralen Ort (dem Briefkasten) erhält - die Web-Billing-Sites potentieller Rechnungssteller regelmäßig abfragen.

\subsection{E-Mail-Billing}

Als E-Mail-Billing bezeichnen wir hier die Übertragung der Rechnung über das Simple-Mail-Transfer-Protocol (SMTP). D.h., der Rechnungsempfänger erhält seine Rechnungen in Form von E-Mails ("E-Bill"; zu einem einfachen Beispiel vgl. Abbildung 2).

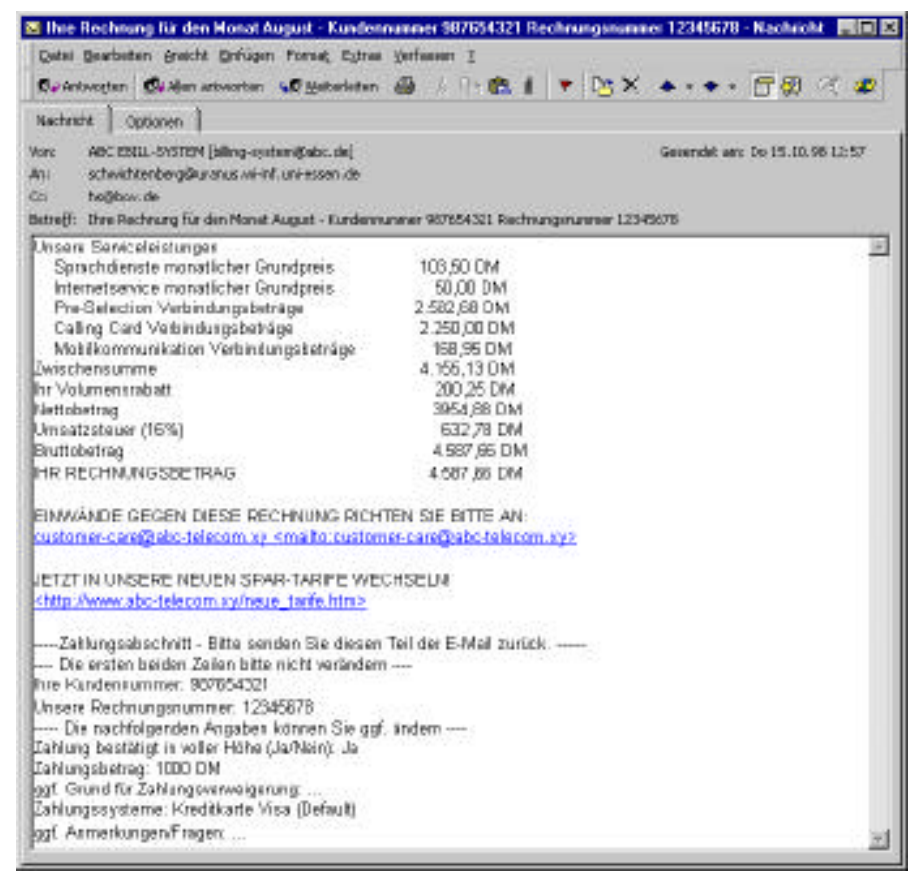

Abbildung 2: Beispiel einer E-Bill mit Zahlungsabschnitt 
Die zugehörigen Zahlungen löst er jeweils durch eine Antwort aus, die die entsprechenden Zahlungsinformationen enthält.

E-Mail-Billing überwindet durch die Verwendung des Information-Push-Konzepts den angesprochenen Nachteil des Web-Billing: Der Kunde erhält seine Rechnungen zentral in seinem E-Mail-Postfach. Allerdings sind im Vergleich zu Web-Bills die Möglichkeiten zur Gestaltung von E-Bills eingeschränkt, da EMails auf reinen ASCII-Texten ohne Formatierung beruhen. Zwar benutzen einige E-Mail-Clients HTML für Rich-Text-Nachrichten; andere verwenden jedoch nur proprietäre Formate oder erlauben keine Formatierungen. Grundsätzlich ist als Ausweg die Übermittlung von Rechnungsdaten in strukturierter Form über Attachments möglich. Allerdings wird in diesem Fall zum einen zusätzliche Software zur Präsentation der Rechnungen benötigt. Zum anderen unterstützen nicht alle verbreiteten E-Mail-Clients das Hyperlinking zwischen Nachrichtentext und Attachments bzw. innerhalb von Attachments, so daß verschiedene Detaillierungsebenen in der E-Bill nicht realisierbar sind. Eine Lösung dieses Problems bestünde in einem Standard für die Informationsverknüpfung in E-Mails, wie er mit der MIME Encapsulation of Aggregate HTML Documents (MHTML) (Palme et al. 1998) vorgeschlagen wird.

Ein weiterer Nachteil des E-Mail-Dienstes besteht darin, daß er nicht die gleiche Interaktivität wie das WWW bietet: Die Interaktion über den Austausch von EMails ist umständlich, erfordert eine starke Strukturierung der Nachrichten und ist vergleichsweise langsam. Die Konfiguration der Rechnungsansicht ist zudem im Vergleich zum Web-Billing unkomfortabel: Der Rechnungssteller muß mit einer E-Bill alle Rechnungs- und Leistungsdaten sowie auch alle zugehörigen Auswertungen verschicken. Die für den Rechnungsempfänger unkomfortable Alternative besteht darin, daß er bestimmte zusätzliche Daten oder Auswertungen durch Senden einer strukturierten E-Mail an den Rechnungssteller bzw. an dessen Server explizit anfordert.

Die Abwicklung des Bill Payment über E-Mail ist grundsätzlich möglich, setzt jedoch strukturierte Nachrichten voraus, deren Korrektheit und Konsistenz mit der Rechnung nicht durch den E-Mail-Client garantiert werden kann. Die Übermittlung und Verarbeitung strukturierter elektronischer Nachrichten ist jedoch im E-Mail-Dienst des Internets noch nicht standardisiert, sondern wird nur von einigen proprietären Systemen wie Lotus Notes und Microsoft Exchange angeboten.

Wie über das Web-Billing kann der Rechnungssteller auch über das E-Mail-Billing ein individuelles Marketing betreiben. Allerdings unterliegt die Werbung, die einer E-Mail beigefügt werden kann, Beschränkungen hinsichtlich des Datenumfangs, da Werbung grundsätzlich nur erfolgreich sein kann, wenn sich der Kunde nicht belästigt fühlt. Zwar kann der Rechnungssteller in seine E-Bills Hyperlinks zu Angeboten im WWW einbinden; die Links haben jedoch nicht die direkte Werbewirkung wie Web-Bills, insbesondere dann nicht, wenn der Rechnungsempfänger zur Kosteneinsparung seine E-Bills offline liest. 
Zusammenfassend kann man somit feststellen, daß sich das E-Mail-Billing - zumindest derzeit - nur für solche Rechnungen eignet, die aus wenigen Detaildaten bestehen und bei denen Auswertbarkeit sowie Marketingaspekte keine bzw. nur untergeordnete Bedeutung besitzen.

\subsection{Integration von Web- und E-Mail-Billing}

Die bisherigen Ausführungen haben gezeigt, daß weder das Web-Billing, noch das E-Mail-Billing in der Lage sind, die Anforderungen an den Rechnungsprozeß allein zu erfüllen. Sinnvoll erscheint daher eine Integration der beiden Dienste, die die Vorteile beider Dienste nutzt und ihre Nachteile vermeidet. Drei Szenarien für eine Integration sollen hier kurz skizziert werden:

\section{Szenario 1: E-Mail-Billing mit Details im Web}

Der Rechnungsempfänger erhält die wesentlichen Bestandteile der Rechnung per E-Mail (Absender, Rechnungsgrund, Betrag, Fälligkeit). Er hat die Möglichkeit, sich Detailinformationen im WWW anzuschauen. Die zugehörigen Zugriffsstellen sind als URLs in der E-Bill enthalten.

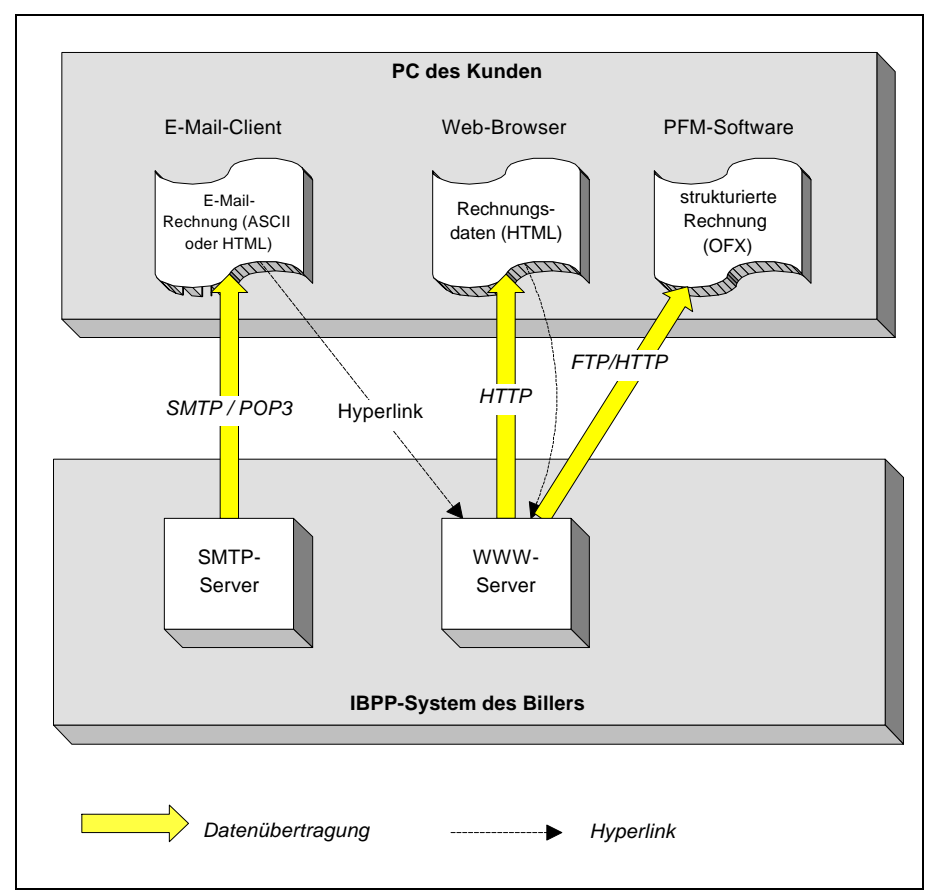

Abbildung 3: E-Mail-Billing mit Details im WWW (Szenario 1) 
Abbildung 3 verdeutlicht das Szenario: Der Rechnungsempfänger erhält vom IBPP-System eine E-Mail mit dem Hinweis auf eine eingegangene Rechnung. In der E-Mail ist ein Hyperlink enthalten, der ihn direkt zu seiner Rechnung auf dem WWW-Server des Rechnungsstellers führt. Er betrachtet dort seine Web-Bill und entschließt sich dann zum Download einer OFX-Datei (je nach vorhandener Konfiguration über HTTP oder FTP), die er in seiner PFM-Software weiterverarbeitet.

\section{Szenario 2: Web-Billing mit E-Mail als Hinweis}

Der Kunde erhält eine E-Mail, sobald im WWW eine neue Rechnung für ihn vorliegt. Die E-Mail enthält die URL, unter der die Rechnung abgerufen werden kann. Alternativ oder ergänzend zur E-Mail können auch andere Medien wie Pager, Telefax oder das Telefon (als Interactive Voice Response System) zur Benachrichtigung des Rechnungsempfängers genutzt werden.

\section{Szenario 3: Web-Billing mit E-Mail als Erinnerung}

Der Kunde wird nur dann per E-Mail über die Rechnung informiert, wenn diese überfällig ist. Auch hier bestehen die genannten Alternativen.

\subsection{Indirektes Internet Bill Presentment and Payment}

Die bisherigen Überlegungen gingen von einer direkten Beziehung zwischen Rechnungssteller und Kunde aus ("direktes Billing"). Von einem indirekten Billing kann gesprochen werden, wenn eine dritte Instanz in den Prozeß eingeschaltet wird. Zwei Konzepte sind für ein solches indirektes Billing zu unterscheiden: Auf der einen Seite kann der Rechnungssteller einen externen Dienstleister beauftragen, die Rechnungen in seinem Auftrag zu versenden. Dieses Konzept, das man als Bill Outsourcing bezeichnen könnte, wird bereits bei papierbasierten Billingprozessen (bspw. im Gesundheitswesen) verwendet. Auf der anderen Seite kann ein (potentieller) Rechnungsempfänger einen Dienstleister damit beauftragen, die Rechnungen verschiedener Rechnungssteller für ihn zu verwalten. Dieses Konzept wird Bill Consolidation (O'Sullivan 1998, S. 52 ff.), der Dienstleister "Bill Consolidator" genannt; alternative Bezeichnungen für das Konzept sind "Bill Concentration" (Roberts 1998) bzw. "Bill Aggregation" (Stonemann 1998, S. 50 ff.).

Bill Consolidation bietet dem Rechnungsempfänger gegenüber dem direkten Billing den Vorteil, daß er eine Kommunikationsbeziehung nur mit einem anstelle von vielen Aktoren unterhalten muß. Dadurch sinken sein Prozeßaufwand und seine Transaktionskosten erheblich. Kreditkartenrechnungen stellen bereits einen Schritt in Richtung der Bill Consolidation dar, fassen allerdings lediglich die Zahlungsvorgänge zusammen.

Abbildung 4 zeigt ein Szenario für die Bill Consolidation auf der Basis des Internets: Der Rechnungssteller übermittelt dem Consolidator die Rechnungsdaten über das Internet. Der Kunde besucht in regelmäßigen Abständen die Website des Consolidator oder wird durch E-Mails des Consolidator auf dessen Website geru- 
fen. Dort erhält er nach einer Authentifizierung eine Übersicht der an ihn addressierten Rechnungen. Mit einer einzigen Transaktion kann der Kunde die Rechnungen an den Consolidator zahlen, der den Gesamtbetrag wiederum aufteilt und den Rechnungsstellern den jeweiligen Rechnungsbetrag überweist.

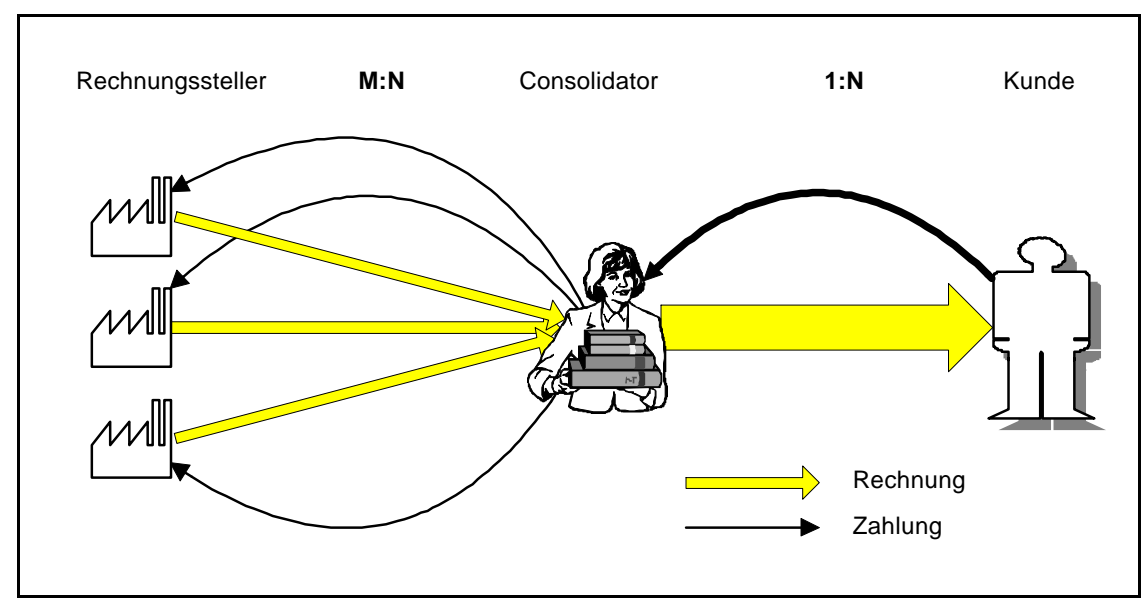

Abbildung 4: Bill Consolidation aus der Sicht eines Kunden

Die Entlohnung eines Bill Consolidator kann grundsätzlich sowohl durch den Kunden als auch durch die Rechnungssteller erfolgen. Denn die Rechnungssteller sparen bei der kundenorientierten Bill Consolidation wie beim Bill Outsourcing die Kosten für eigene IBPP-Server ein.

Prädestiniert als Anbieter von Bill Consolidation-Diensten sind Banken, da sie als Finanzdienstleister bereits im Auftrag von Privathaushalten und Unternehmen tätig sind. Insbesondere stellt die Bill Consolidation eine gute Ergänzung zum Internet Banking dar: Der Kunde erhält bei einer solchen Kombination nicht nur seine Rechnungsabwicklung, sondern auch die Abwicklung seiner Bankgeschäfte aus einer Hand. Die Bank kann damit ihre Verbindung zum Kunden stärken. Weitere geeignete Anbieter einer Bill Consolidation sind Kreditkartenunternehmen sowie grundsätzlich auch Softwarehersteller und Telekommunikationsunternehmen, da sie über die entsprechende Internet-Plattform verfügen. Neben seinen Vorteilen beinhaltet das Bill Consolidation-Modell allerdings auch Nachteile: Der Rechnungssteller gibt die Kundenschnittstelle an den Bill Consolidator ab, der sie selbst vermarkten kann. Aus der Sicht des Kunden stehen dem hohen Komfort Einschränkungen bei den Analysemöglichkeiten als Nachteil gegenüber, weil der Consolidator i.a. nicht die gleiche Datenbasis und die gleichen Analysewerkzeuge wie der Rechnungssteller vorhalten kann. Vorgeschlagen wird aus diesen Gründen ein sog. Thin-Consolidation-Model (vgl. Abbildung 5); es sieht vor, daß jeweils nur die 
Kerndaten der Rechnung an den Consolidator übertragen werden, die Detaildaten dagegen auf dem Server des Rechnungsstellers verbleiben (Just in Time Solutions 1998).

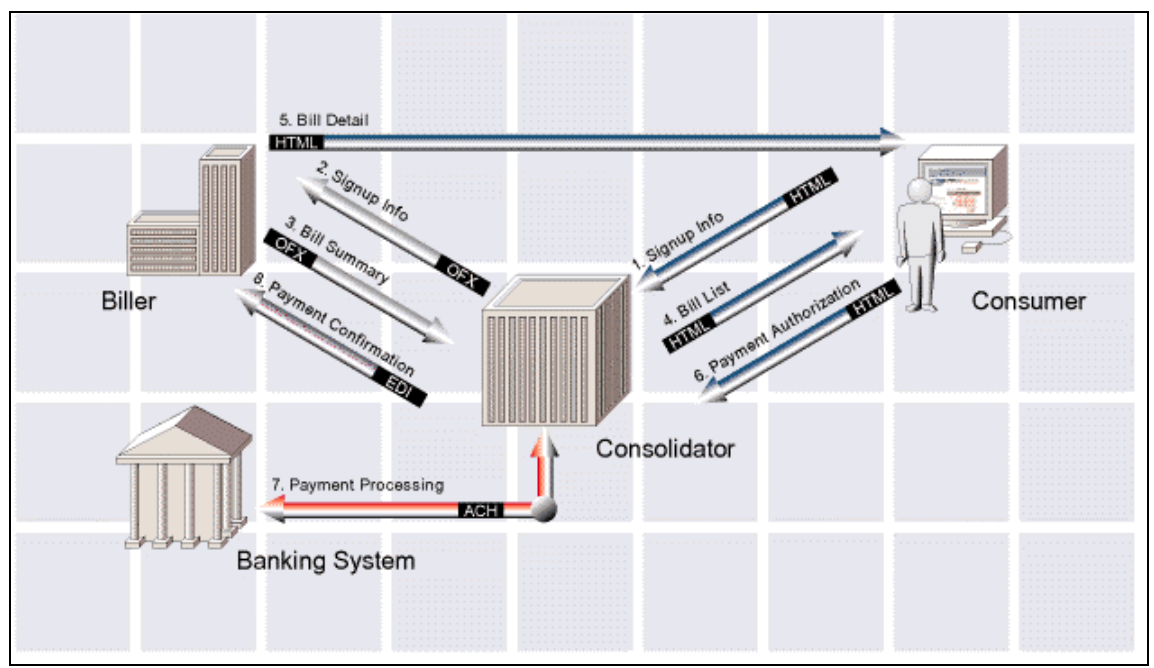

Abbildung 5: Thin Consolidation bei Just In Time Solutions (Just in Time Solutions 1998)

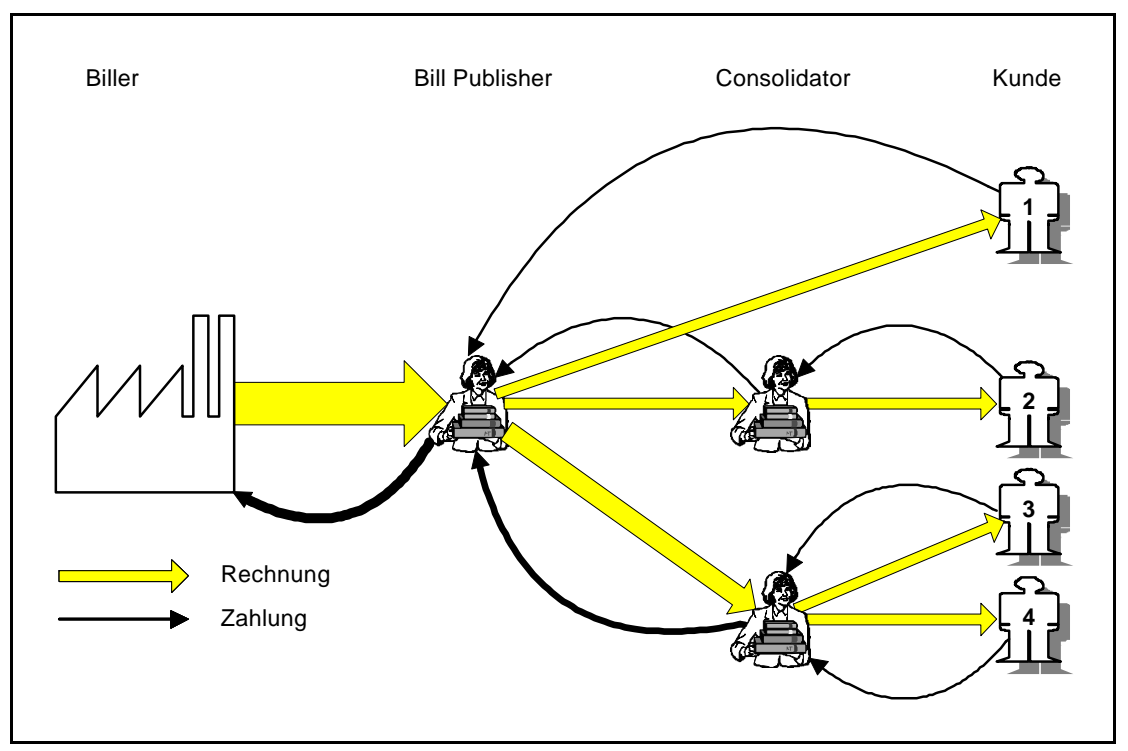

Abbildung 6: Bill Outsourcing und Bill Consolidation - kombiniertes Modell 
Schließlich können auch Bill Consolidation und Bill Outsourcing miteinander kombiniert werden. Abbildung 6 stellt dieses kombinierte Modell dar.

\subsection{Rechtsfragen des Electronic Billing}

Die Rechtsprechung bejaht heute weitgehend, daß durch Computersysteme automatisch erzeugte und per Datenübertragung übermittelte Willensäußerungen rechtlich ordnungsgemäß sind (Killian 1996, S. 111 ff.; Strömer 1997, S. 86). Gemäß §126 des Bürgerlichen Gesetzbuches (BGB) ist für Willenserklärungen und Verträge ein Papierbeleg entbehrlich, sofern nicht ein Gesetz die Schriftform explizit vorschreibt (Schuppenhauer 1994, S. 2041). Da das BGB keine Regelungen für die Rechnung vorsieht, ist der elektronische Versand von Rechnungen im Geschäftsverkehr möglich. Auch das Handelsgesetzbuch (HGB) stellt kein Hindernis dar. Es kann als "zeitgemäß" bezeichnet werden, da §239 Abs. 3 die Speicherung von Büchern und Aufzeichnungen auf Datenträgern sowie \$257 Abs. 3 die bildliche Aufbewahrung von Belegen auf Datenträgern gestattet (Schuppenhauer 1994, S. 2041).

Für die elektronische Rechnung ergeben sich dennoch rechtliche Probleme. Betroffen ist vor allem der Rechnungssteller, da er bei Ausbleiben der Zahlung beweisen muß, daß die Rechnung dem Empfänger zugegangen ist (Palandt 1998, $\S 130$, Randnummer 21). Eine Rechnung gilt dann als zugegangen, wenn sie in den "Machtbereich" des Empfängers gelangt ist (Strömer 1997, S. 89); bei der Briefpost stellt der Briefkasten den Machtbereich des Empfängers dar, bei elektronischer Post analog das E-Mail-Postfach auf dem SMTP-Server des Internet-Providers des Empfängers. Von der Rechtsprechung wird ein Geschäftspartner, der seine E-Mail-Adresse veröffentlicht, wie der Empfänger normaler Post behandelt, d.h. es wird erwartet, daß er sein E-Mail-Postfach mindestens einmal täglich leert (Strömer 1997, S. 90; OLG Köln 1989). Übermittlungsfehler, die auf ein Fehlverhalten seines Providers zurückzuführen sind, hat der Empfänger zu vertreten (ebenda).

Die offene Frage ist, wie der Rechnungssteller den Zugang einer E-Bill im Postfach des Empfängers (nachträglich) beweisen kann. Die Rechtsprechung erkennt bei der Telefaxübertragung das Sendeprotokoll nicht als Beweis an. Dies gilt leider analog für das Sendeprotokoll einer E-Mail, da beim E-Mail-Transport - wie bei der Telefaxübertragung - zahlreiche potentielle Fehlerquellen existieren. Vorgeschlagen wird zur Beweissicherung die Bestätigung elektronisch übermittelter Dokumente (Schuppenhauer 1994, S. 2044; Strömer 1997, S. 88). Als Beweis für den Zugang elektronischer Rechnungen könnte entsprechend eine vom Kunden gesendete E-Mail gelten. Dazu müßte sich ein Verfahren etablieren, um die Authentizität und Integrität einer elektronischen Nachricht sicherzustellen. Die Sendung der Mail müßte durch eine entsprechende Funktion automatisiert werden. Alternativ kann das Problem durch eine in das TCP/IP-Protokoll integrierte Routine gelöst werden, die die korrekte und vollständige Übermittlung der E-Bill 
bis zur Schnittstelle des Empfängers zuverlässig und nicht manipulierbar für den Absender aufzeichnet.

Wie die Rechtsprechung den Machtbereich des Empfängers beim Web-Billing definieren wird, ist noch unklar. Sicherlich kann jedoch der Rechnungssteller seiner Benachrichtigungspflicht nicht dadurch nachkommen, daß er eine Rechnung auf der IBPP-Website bereitstellt und es dem Kunden überläßt, die Site regelmäBig abzufragen. Von daher können aus rechtlicher Sicht - zumindest auf absehbare Zeit - nur die oben skizzierten Szenarien für eine Integration von EBilling und Web-Billing realisiert werden.

Ungewiß ist allerdings auch noch, welchen Beweiswert elektronisch erzeugte, gesendete und archivierte Nachrichten vor Gerichten tatsächlich besitzen. Die Zivilprozeßordnung (ZPO) in $§ 416$ fordert im Rechtsstreit den formellen Beweis durch eine Urkunde in Gestalt des Papieroriginals (Schuppenhauer 1994, S. 2041). Die Rechtsprechung anderer Staaten ist in diesem Punkt sehr viel fortschrittlicher: In den USA sind digitale Unterschriften bereits juristisch bindend (Lynch/Lundquist 1997, S. 206), in Großbritannien, Kanada und den USA sogar unsignierte E-Mails als Beweismittel zugelassen (Strömer 1997, S. 92).

Rechnungen bzw. der Zugang von Rechnungen sind nicht nur vor Gericht, sondern auch für das Finanzamt von Interesse. Die deutschen Finanzbehörden erkennen jedoch nur Schriftstücke an, die auf der Seite des Rechnungsstellers ausgedruckt wurden; Ausnahmen sind nur auf Microfiche gespeicherte sowie per Telefax oder Telex übertragene Schriftstücke (BMF 1992, S. 2). Elektronische Rechnungen werden nur anerkannt, wenn zusätzlich eine schriftliche Leistungsabrechnung vorliegt. Diese schriftliche Abrechnung kann in Form von Protokollen über die übertragenen Dateiinhalte oder durch entsprechende Dateiausdrucke erfolgen. Die Zusammenfassung von elektronischen Rechnungen zu schriftlichen Sammelabrechnungen ist möglich.

Probleme bereitet die fehlende Anerkennung elektronischer Rechnungen durch die Finanzbehörden zum einen dem Privatkunden beim Nachweis von Werbungskosten, Vorsorgeaufwendungen, außergewöhnlichen Belastungen etc. im Rahmen seiner Einkommenssteuererklärung und zum anderen den Unternehmen wegen des Vorsteuerabzugs. Das Problem der Privatkunden kann durch eine zusammenfassende schriftliche Jahresabrechnung gelöst werden. Auch der Vorsteuerabzug ist auf der Basis einer Sammelabrechnung möglich. Der Geschäftskunde benötigt somit zeitnah - i.d.R monatlich oder vierteljährig - eine schriftliche Sammelrechnung, da er sonst Liquidität verliert. 


\section{Konzeption eines IBPP-Systems für ein Telekommunikationsunternehmen}

\subsection{Architektur}

Abbildung 7 zeigt einen Entwurf für die Architektur eines IBPP-Systems, das für ein großes Telekommunikationsunternehmen konzipiert wurde. Das System umfaßt Web- und E-Mail-Billing, Bill Consolidation-Unterstützung sowie eine $\mathrm{Cu}$ stomer Care-Schnittstelle.

Die Grundlage des Systems bildet eine Datenbank mit den Rechnungs- und Leistungsdaten, den Daten des Rechnungswesens sowie den Profilen der Kunden. Letztere bestehen nicht nur aus den zur Erstellung der Rechnung erforderlichen Stammdaten wie Name und Adresse, sondern enthalten auch demographische Daten sowie Informationen zum bisherigen Kaufverhalten, die als Basis des OneTo-One-Marketing benötigt werden.

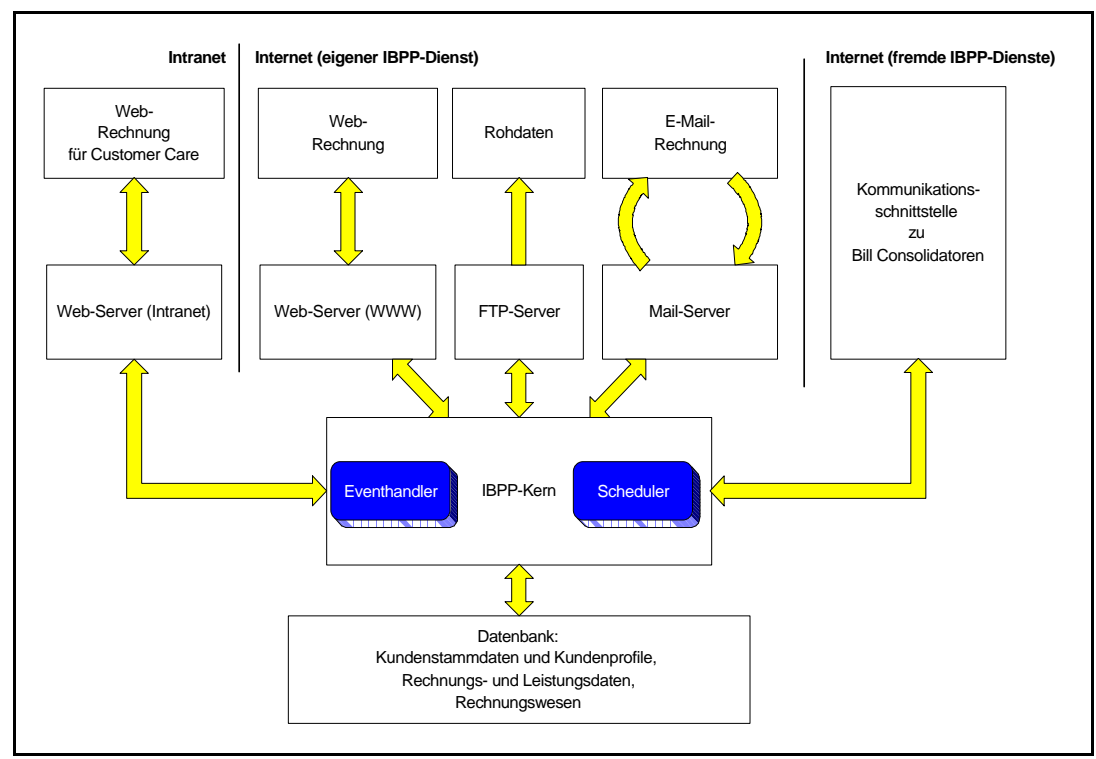

Abbildung 7: Architektur eines IBPP-Systems

Als Präsentationskomponenten sieht die Architektur zwei Web-, einen FTP- und einen Mail-Server sowie die Verbindungen zu den IBPP-Systemen von Bill Consolidators vor. Einer der beiden Web-Server ist mit dem Internet verbunden; er gestattet den Kunden den Zugriff auf ihre Web-Bills. Der andere Web-Server steht im Intranet des Unternehmens und bietet den Customer Care-Mitarbeitern 
Zugriff auf die Rechnungen. Der FTP-Server ermöglicht den Download von Rechnungs- und Leistungsdaten in verschiedenen Formaten. Der SMTP-Server schließlich dient zum Versand von E-Bills.

Im Mittelpunkt der Architektur steht ein Kernsystem, das die Daten aus der Datenbank entnimmt, aufbereitet und über die Präsentationskomponenten zur Verfügung stellt. Das Kernsystem besitzt einen Eventhandler, der auf Ereignisse reagiert, die über die Präsentationskomponenten eingehen. Dabei handelt es sich beim Web-Server um Anfragen nach einer Seite (inklusive der übergebenen Parameter) und beim Mail-Server um eingehende E-Mails. Außerdem sieht die Architektur vor, daß der Kunde durch strukturierte E-Mail-Nachrichten Anfragen an das System stellen kann (z.B. Zahlungen bestätigen, Reklamationen einreichen, Auswertungen anfordern etc.). Der Kern nimmt die Anfragen entgegen und generiert bei Bedarf eine Antwort durch Zugriff auf die Datenbasis (z.B. Eingangsbestätigung für Zahlung oder Reklamation, angeforderte Auswertung). Er ist in der Lage, alle gängigen Datenformate zu generieren (HTML, GIF, JPEG, OFX, GOLD, Excel etc.). Ein Scheduler dient der Ausführung von Anfragen, die nicht zur sofortigen Abarbeitung gestellt, sondern mit einem Erledigungstermin versehen wurden. Bei solchen terminierten Anfragen handelt es sich beispielsweise um Zahlungen eines Kunden, die erst zu einem bestimmten Datum ausgeführt werden sollen, um Liquiditätsvorteile zu realisieren. Schließlich enthält die Architektur eine Kommunikationsschnittstelle zu Bill Consolidatoren.

\subsection{Bill Presentment der Telekommunikationsrechnung}

Aus dem entworfenen Konzept für das Bill Presentment der Telekommunikationsrechnung sollen im folgenden einige Beispiele vorgestellt werden, um die Möglichkeiten des Bill Presentment zu illustrieren (vgl. Abbildung 8). 


\begin{tabular}{|c|c|c|}
\hline \multicolumn{2}{|l|}{ Ihre Rechnung für August 1998} & ABC Telecom \\
\hline \multicolumn{2}{|l|}{ ABC telecom } & $\begin{array}{l}\text { Rechnungsdatum } \\
30.7 .1998\end{array}$ \\
\hline \multicolumn{2}{|l|}{ Firmenbezeichnung 1} & \multirow[t]{2}{*}{$\begin{array}{l}\text { Kundennummer } \\
1234567890\end{array}$} \\
\hline \multicolumn{2}{|l|}{ Firmenbezeichnung 2} & \\
\hline \multirow{2}{*}{\multicolumn{2}{|c|}{$\begin{array}{l}\text { Firmenbezeichnung } 3 \\
\text { Straße / Postfach }\end{array}$}} & \multirow{2}{*}{$\begin{array}{l}\text { Rechnungsnummer } \\
0987654321\end{array}$} \\
\hline & & \\
\hline PLZ Ort-Stadtteil & & \\
\hline \multicolumn{2}{|c|}{$\begin{array}{l}\text { Vereinbarungsgemäß werden wir diesen Betrag am 15.9.1998 } \\
\text { von Ihrem Konto } 12345678 \text {, BLZ } 123456 \text { abbuchen. Vielen Dank! }\end{array}$} & $\begin{array}{l}\text { Klicken Sie aut die Serviceleistungen, } \\
\text { um Details zu erhalten }\end{array}$ \\
\hline Unsere Serviceleistungen & Betrag DM & \\
\hline Sprachdienste monatlicher Grundpreis & $103,50 \mathrm{DM}$ & \\
\hline Internetservice monatlicher Grundpreis & $50,00 \mathrm{DM}$ & \\
\hline Pre-Selection Verbindungsbeträge & $2.582,68 \mathrm{DM}$ & Graphisch darstellen \\
\hline Calling Card Verbindungsbeträge & $1.250,00 \mathrm{DM}$ & Download \\
\hline Mobilkommunikation Verbindungsbeträge & $168,95 \mathrm{DM}$ & \\
\hline Zwischensumme & $4.155,13 \mathrm{DM}$ & \\
\hline Ihr Volumensrabatt & 200,25 & $\underline{\text { Service }}$ \\
\hline Nettobetrag & $3.954,88 \mathrm{DM}$ & \\
\hline Umsatzsteuer (16\%) & $632,78 \mathrm{DM}$ & \\
\hline Bruttobetrag & $4.587,66 \mathrm{DM}$ & zurück \\
\hline Ihr Rechnungsbetrag & $4.587,66 \mathrm{DM}$ & zur Rechnungsübersicht \\
\hline
\end{tabular}

Abbildung 8: Telekommunikationsrechnung als Web-Bill

Charakteristisch für Telekommunikationsrechnungen sind ihre große Anzahl sowie die zu einer Rechnung gehörenden umfangreichen Detaildaten, die als Einzelverbindungsnachweise (EVN) dargestellt werden. Das IBPP-System läßt dem Rechnungsempfänger die Wahl, in welcher Weise Web-Billing, E-MailBilling oder eine Kombination aus beiden eingesetzt werden soll. Beispielsweise kann er festlegen, daß er die Rechnung und den EVN komplett per E-Mail erhält oder alternativ nur die Rechnung mit einem Hyperlink auf den EVN in der WebBilling-Site. 


\begin{tabular}{|c|c|c|c|c|c|c|}
\hline \multicolumn{5}{|c|}{ Einzelverbindungsnachweis } & \multicolumn{2}{|c|}{ ABC Telecom } \\
\hline für Nebenstelle & \multicolumn{6}{|c|}{01234 / 123456} \\
\hline von: & 01.08.1998 & \multicolumn{5}{|l|}{$00: 00$} \\
\hline \multicolumn{2}{|r|}{25.08 .1998} & \multicolumn{5}{|l|}{$12: 00$} \\
\hline Datum & Beginn & $\underline{\text { Dauer }}$ & $\underline{\text { Rufnummer }}$ & $\begin{array}{r}\text { Zeit } \\
\underline{\text { Zone }} \\
\end{array}$ & Enf.-Zone & Preis * \\
\hline 07.08 .1998 & $00: 05$ & $00: 05: 20$ & 001408253723 & $\mathrm{NZ}$ & Welt 1 & $4,80 \mathrm{DM}$ \\
\hline 05.08 .1998 & $09: 15$ & 00:00:56 & 08964162323 & $\mathrm{HZ}$ & National & $0,33 \mathrm{DM}$ \\
\hline 06.08 .1998 & $10: 22$ & 00:02:10 & 0693452323 & $\mathrm{HZ}$ & National & $0,76 \mathrm{DM}$ \\
\hline 09.08 .1998 & $15: 43$ & 01:20:47 & 02355642234 & $\mathrm{HZ}$ & Regional & $16,16 \mathrm{DM}$ \\
\hline 05.08 .1998 & $17: 02$ & 00:03:31 & 0892345123 & $\mathrm{HZ}$ & National & $1,23 \mathrm{DM}$ \\
\hline \multirow[t]{2}{*}{ Summe } & & 01:32:44 & \multirow{2}{*}{\multicolumn{4}{|c|}{\begin{tabular}{l|r} 
& 23,27 DM \\
* ohne Volumensrabatte
\end{tabular}}} \\
\hline & & & & & & \\
\hline \multicolumn{3}{|c|}{$\begin{array}{l}\text { Darstellungsoptionen: } \\
\text { Spaltenauswahl }\end{array}$} & $\begin{array}{l}\text { Zustellung: } \\
\text { Alles Drucken }\end{array}$ & & \multicolumn{2}{|c|}{$\begin{array}{l}\text { Weitere Funktionen: } \\
\text { Statistiken }\end{array}$} \\
\hline \multicolumn{3}{|l|}{ Sortierung } & Download & & \multirow{2}{*}{\multicolumn{2}{|c|}{$\begin{array}{l}\text { weiter zur nächsten Seite } \\
\text { zurück zur Selektion }\end{array}$}} \\
\hline \multicolumn{3}{|c|}{$\begin{array}{l}\text { Gruppierung } \\
\text { Zwischensummen }\end{array}$} & \multicolumn{2}{|l|}{ E-Mail } & & \\
\hline
\end{tabular}

Abbildung 9: Einzelverbindungsnachweis im WWW

Im Rahmen des Web-Billing stehen dem Kunden verschiedene Detaillierungsebenen zur Verfügung. Im Bereich "Kontostand" sieht der Kunde die Bewegungen auf seinem Kundenkonto: Rechnungen, Einzahlungen und Gutschriften. Von jeder Rechnung (vgl. noch einmal Abbildung 8) führt ein Link zu einer detaillierten Rechnungsdarstellung, die die verschiedenen in Anspruch genommenen Leistungen (z.B. Kommunikation Festnetz, Kommunikation Mobil, Calling Card, Internet) auflistet. Von dort wiederum führt jeweils ein Link zur entsprechenden Einzelverbindungsansicht (vgl. Abbildung 9). In dieser Ansicht kann der Kunde die Darstellung selbst gestalten: Eine Sortierung der Daten ist nach allen verfügbaren Spalten (z.B. Datum, Uhrzeit, Anschlußnummer, Zielrufnummer, Zielort, Tarif, Dauer, Kosten) möglich, Spalten sind ein- und ausblendbar und können für Gruppierungen genutzt werden (vgl. Abbildung 10). Geschäftskunden können für weitere Auswertungen Organisationseinheiten in das System einpflegen und bestimmten Anschlußnummern zuordnen. Dem weniger versierten Kunden werden außerdem verschiedene Standardberichte angeboten.

Auswertungen können insbesondere auch über die Grenzen einer Rechnung hinweg erstellt werden. Dadurch wird zum einen berücksichtigt, daß der Abrechnungszeitraum des Telekommunikationsanbieters nicht zwangsläufig mit dem des Kunden übereinstimmt. Außerdem können zum anderen Veränderungen der Kosten im Zeitablauf untersucht werden. Hinderlich ist hier allerdings die Telekommunikationsdienstunternehmen-Datenschutzverordnung (TDSV). Sie verlangt, daß der Telekommunikationsanbieter die Verbindungsdaten nach maximal 80 Tagen löscht. Insbesondere deshalb ist ein Download der Daten möglich. 


\section{Statistik}

Verkehrsmessung Wochentage

$\begin{array}{lll}\text { für: } & \text { Kostenstelle Einkauf } & \\ \text { von: } & 01.08 .1998 & 00: 00 \\ \text { bis: } & 25.08 .1998 & 12.00\end{array}$

\begin{tabular}{|c|c|c|c|c|}
\hline Nr. & Wochentag & $\begin{array}{c}\text { Anzahl } \\
\text { Gespräche }\end{array}$ & $\begin{array}{c}\text { Durchschn. } \\
\text { Gesprächslänge }\end{array}$ & Preis * \\
\hline
\end{tabular}

\begin{tabular}{|c|c|c|c|c|}
\hline 1 & Montag & 251 & 2,8 Minuten & $562,24 \mathrm{DM}$ \\
\hline 2 & Dienstag & 180 & 2,1 Minuten & $302,40 \mathrm{DM}$ \\
\hline 3 & Mittw och & 179 & 2,1 Minuten & $300,72 \mathrm{DM}$ \\
\hline 4 & Donnerstag & 169 & 2,3 Minuten & $310,96 \mathrm{DM}$ \\
\hline 5 & Freitag & 110 & 3,0 Minuten & $264,00 \mathrm{DM}$ \\
\hline 6 & Samstag & 3 & 4,0 Minuten & $9,60 \mathrm{DM}$ \\
\hline 7 & Sonntag & 5 & 4,2 Minuten & $16,80 \mathrm{DM}$ \\
\hline & & & & $1.766,72 \mathrm{DM}$ \\
\hline
\end{tabular}

graphisch darstellen

zurück

Abbildung 10: Auswertung der Tk-Gebühren nach Wochentagen

Die mangelnde Beweiskraft elektronisch übermittelter Rechnungen vor Gericht wird z.T. dadurch kompensiert, daß Zahlungserinnerungen und Mahnungen auf dem traditionellen Postweg versendet werden. 


\section{Literaturverzeichnis}

Ahuja, V. (1996): Network and Internet Security. Boston 1996.

BMF (1992): Anerkennung der Rechnungsstellung durch Telefax, Telex, Datenfernübertragung oder Datenträgeraustausch als Rechnung im Sinne $\$ 14$ UStG”. Bundessteuerblatt, Heft 8, 1992, S. 376-379.

COBWEB (1998): A Compendium of Electronic Commerce Terms. http://www. merchantgateway.com/glossary.htm, Abruf am 14.6.1998.

Craft, G.R./Johnson, W. (1998): The Emerging Electronic Bill Presentment Industry - A Tale of Two Camps. White Paper der Firma Robertson, Stephens \& Company, http://www.edocs.com/Reports/Craft897.pdf, Abruf am 14.7.98.

CyberCash (1998): CyberCash Interactive Billing and Payment. http://www.cybercash.com/cybercash/billers/, Abruf am 16.7.98.

Dittrich, T. (1998): HFN Research - Updated Electronic Bill Presentment Study, Studie des Home Financial Network, Stand 24.3.1998, http://www. homenetwork.com/articles/res980300.html, Abruf am 10.6.1998.

Geml, R./Geisbüsch, H.-G./Lauer, H. (1997): Das kleine Marketing-Lexikon. Düsseldorf 1995.

Illingworth, V. et al. (1997): Oxford Dictionary of Computing. Oxford 1997.

Integrion Financial Network (1998): Gold Standard Message Transaction Specification Release 2.8.4, Fourth Edition (Januar 1998), http://www. integrion.net/gold/CHP1TO6.pdf, Abruf am 5.6.1998.

Just in Time Solutions (1998): Internet Billing Architectures, http://www. justintime.com/why/intbill/architecture.html, Abruf am 8.6.1998.

Kalakota, R./Whinston, A. B. (1996): Frontiers of Electronic Commerce. New York et al. 1996.

Kilian, W./Heusser, B. (Hrsg.) (1996): Computerrechtshandbuch. Computertechnologie in der Rechts- und Wirtschaftspraxis. Baden-Baden 1996.

Lynch, D.C./Lundquist, L. (1997): Zahlungsverkehr im Internet, München et al. 1997.

Mehnen, H. (1989): Telekommunikation in Handel, Transport und Verwaltung nach internationalem Standard - ISO-Standard 9735 EDIFACT. Eschborn 1989.

Mitchell, R. (1997): Still a dream. In: Credit Card Management, Heft 10, 1997, S. 65-66.

Nieschlag, R./Dichtl, R./Hörschgen, H. (1988): Marketing, Berlin 1988.

Nusser, S. (1998): Sicherheitskonzepte im WWW. Berlin 1998. 
OLGKöln (1998): Zugang von Willenserklärungen. Entscheidungssammlung Online-Recht OLG Köln 891201, http://www.online-recht.de/vorent. html?OLGKoeln891201+ref=Vertragsrecht, Abruf am 12.8.1998.

Open Financial Exchange (1998a): Bill Presentment, Open Financial Exchange Homepage. http://www.ofx.net/ofx/i_bill.asp, Abruf am 14.5.1998.

Open Financial Exchange (1998b): Open Financial Exchange Specification. Open Financial Exchange Homepage, ftp://ftp.qfn.com/pub/corporate/ofex change/ofx15s.pdf, Abruf am 12.8.1998.

Orr, B. (1997): Electronic bill paying shows signs of soaring. ABA Banking Journal, Heft 5, 1997 (zitiert nach http://www.banking.org/aba/bank tech0597.htm. Abruf am 12.8.1998).

O'Sullivan, O. (1998): The check is in the e-mail. ABA Banking Journal, Heft 1, 1998, S. 52-55.

Palandt, O. (1998): Bürgerliches Gesetzbuch, München 1998.

Palme, J. et al. (1998): MIME Encapsulation of Aggregate Documents. Internet Draft, Stand Februar 1998, http://www.ietf.org/internet-drafts/draft-ietfmhtml-rev-06.txt, Abruf am 12.8.1998.

Roberts, B. (1998): Billers Pave Way for Web Payments, But Consumers Are Leery. Internet World, Heft 1, 1998 (zitiert nach http://www. internetworld.com/print/1998/01/12/ecomm/1990112-pave.html, Abruf am 12.8.1998).

Schulzki-Haddouti, C. (1998): Grobschnitt - Das Online-Recht muß nachgebessert werden. c't - Magazin für Computertechnik, Heft 16, 1998, S. 160-161.

Schuppenhauer, R. (1994): Beleg und Urkunde - ganz ohne Papier. Der Betrieb, Heft 41, 1994, S. 2041-2047.

Stahlknecht, P. (1993): Einführung in die Wirtschaftsinformatik. Berlin 1993.

Stoetzer, M.-W. (1994): Neue Telekommunikationsdienste: Stand und Perspektiven in der deutschen Wirtschaft. Ifo-Schnelldienst, Heft 7, 1994, S. 8-19.

Stoneman, B. (1998): Treading cautiously. Banking Strategies, Heft 2, 1998, S. 50-54.

Strömer, T.H. (1997): Online-Recht. Heidelberg 1997.

Talmar, S. (1998): Pioneers in e-commerce. The Banker, Heft 1, 1998, S. 69-71.

Zwischenberger, R. (1998): Einführung in UB/EDIFACT. Fachhochschule Salzburg, http://www.tks.fh-sgb.ac.at/ rzwischen/edifact.html, Abruf am 14.7.1998. 